PROCEEDINGS OF THE

AMERICAN MATHEMATICAL SOCIETY

Volume 125, Number 7, July 1997, Pages 2029-2032

S $0002-9939(97) 03808-2$

\title{
HÖLDER CONTINUITY PROPERTY OF FILLED-IN JULIA SETS IN $\mathbb{C}^{n}$
}

\author{
MARTA KOSEK
}

(Communicated by Eric Bedford)

\begin{abstract}
It is proved that the pluricomplex Green function of the filled-in Julia set $J$ associated with a polynomial mapping in $\mathbb{C}^{n}$ is Hölder continuous. This yields in particular that $J$ preserves Markov's inequality.
\end{abstract}

\section{INTRODUCTION}

Different classes of Julia sets have been already studied by several authors (see $[\mathrm{CG}]$ and the references given there). In this paper following M.Klimek [K1] we will consider the filled-in Julia sets defined as follows:

Let $P: \mathbb{C}^{n} \longrightarrow \mathbb{C}^{n}$ be a polynomial mapping satisfying

$$
\liminf _{|z| \rightarrow \infty} \frac{|P(z)|}{|z|^{\delta}}>0
$$

with some $\delta>1$. We define the filled-in Julia set associated with $P$ to be the set

$$
J_{P}:=\left\{z \in \mathbb{C}^{n}:\left\{P^{j}(z)\right\}_{j \in \mathbb{N}_{0}} \text { is bounded }\right\},
$$

where $P^{j}$ denotes the $j$-th iteration of the polynomial mapping $P$.

We are interested in potential-theoretic properties of these sets. Let us recall the definition of the $L$-extremal function in $\mathbb{C}^{n}$. For a compact subset $E$ of $\mathbb{C}^{n}$ the function

$$
V_{E}:=\sup \left\{u: u(z) \leq \beta+\log ^{+}|z| \text { for } z \in \mathbb{C}^{n} \text { and } u \leq 0 \text { on } E\right\}
$$

is called the $L$-extremal function corresponding to $E$. A compact set $E$ in $\mathbb{C}^{n}$ is said to be $L$-regular if $V_{E}$ is continuous. It is well-known that $V_{E}$ is a multidimensional counterpart of the classical Green function. For background material and thorough bibliography we refer the reader to [S2] or [K2].

In [K3] M.Klimek proved the following theorem, which answers the question about the continuity of the $L$-extremal function corresponding to a filled-in Julia set:

Theorem 1.1 ([K3, Corollary 6$])$. Let $P: \mathbb{C}^{n} \longrightarrow \mathbb{C}^{n}$ be a polynomial mapping satisfying (1.1) with some $\delta>1$. Then the filled-in Julia set $J_{P}$ associated with $P$ is compact, polynomially convex and L-regular. Moreover

$$
P^{-1}\left(J_{P}\right)=J_{P}=P\left(J_{P}\right) \text {. }
$$

Received by the editors September 27, 1995 and, in revised form, January 23, 1996.

1991 Mathematics Subject Classification. Primary 32F05, 31C10.

This research was supported by KBN Grant No. 956/P03/95/08.

(C)1997 American Mathematical Society 
In this paper we show that it actually is Hölder regular, i.e. the following theorem holds:

Theorem 1.2. Let $P: \mathbb{C}^{n} \longrightarrow \mathbb{C}^{n}$ be a polynomial mapping satisfying (1.1) with some $\delta>1$. Then there exist constants $C>0$ and $\alpha \in(0,1]$ such that

$(\mathrm{HCP})$

$$
V_{J_{P}}(z) \leq C\left[\operatorname{dist}\left(z, J_{P}\right)\right]^{\alpha}, \quad \text { if } \operatorname{dist}\left(z, J_{P}\right) \leq 1 .
$$

In other words, Theorem 1.2 shows that the pluricomplex Green function $V_{J_{P}}$ of the filled-in Julia set $J_{P}$ has a Hölder continuity property.

The corresponding theorem in the case $n=1$ is due to N.Sibony (1981). For the proof of that fact for quadratic polynomials we refer the reader to [CG, Theorem 8.3.2]. Our proof is based on a similar idea. This result was also already known for certain polynomial mappings on $\mathbb{C}^{2}$. J.E.Fornaess and N.Sibony studied properties of Julia sets associated with complex Hénon mappings (see [FS]). They obtained Hölder continuity of the $L$-extremal function corresponding to a set from this class (see [FS, Theorem 1.2]) and they mentioned that the proof easily extends to some bigger family of generalized Hénon mappings.

Recently A.Edigarian [E] found a nice generalization of Theorem 1.2; namely he proved that every $L$-regular compact set $E$ in $\mathbb{C}^{n}$ has the Hölder continuity property, if there exist a $C^{\infty}$-mapping $F$ and a $\delta>1$ such that $V_{E}(F(z)) \geq \delta V_{E}(z)$ for $z \in \mathbb{C}^{n}$.

We say that a set $E$ in $\mathbb{C}^{n}$ preserves Markov's inequality if for each polynomial $p$

$$
|\operatorname{grad} p(z)| \leq M(\operatorname{deg} p)^{m} \sup _{E}|p|, \quad \text { for } z \in E,
$$

where $M$ and $m$ are positive constants depending only on $E$. Since A. A. Markov proved (M) for $E=[-1,1] \subset \mathbb{C}$ in 1889 (see [M]), it has played an important role in the developing of the constructive theory of functions and has become the object of extensive research (see $[\mathrm{RS}]$ in the one-dimensional case, $[\mathrm{PP}]$ for $\mathbb{R}^{n}$ and $[\mathrm{P}]$ for $\left.\mathbb{C}^{n}\right)$.

It is well-known that the (HCP) property is sufficient for $E$ to preserve Markov's inequality ([S1, Remark after Lemma 1]). Hence by Theorem 1.2 we immediately get

Corollary 1.3. Let $P: \mathbb{C}^{n} \longrightarrow \mathbb{C}^{n}$ be a polynomial mapping satisfying (1.1) with some $\delta>1$. Then the filled-in Julia set $J_{P}$ associated with $P$ satisfies Markov's inequality.

\section{Preliminaries}

From now on, $P$ denotes a polynomial mapping of degree $d$ satisfying (1.1) with some $\delta>1$.

Most of the material in this paper relies on the following transformation rule for the $L$-extremal functions (see [K2, Theorem 5.3.1]):

Theorem 2.1 (M. Klimek). Let $E$ be a subset of $\mathbb{C}^{n}$. Then

$$
\frac{1}{d}\left(V_{E} \circ P\right) \leq V_{P^{-1}(E)} \leq \frac{1}{\delta}\left(V_{E} \circ P\right) .
$$

Let us mention the following trivial consequences of (2.1):

$$
\frac{1}{d^{j}}\left(V_{E} \circ P^{j}\right) \leq V_{P^{-j}(E)} \leq \frac{1}{\delta^{j}}\left(V_{E} \circ P^{j}\right) \quad \text { for } j \in \mathbb{N}_{0}
$$


and of (1.2):

$$
P^{-j}\left(J_{P}\right)=J_{P}=P^{j}\left(J_{P}\right) \quad \text { for } j \in \mathbb{N}_{0} .
$$

Here $P^{-j}(E)$ denotes $\left(P^{j}\right)^{-1}(E)$.

The following consequence of (1.1) will be needed in Section 3.

Lemma 2.2 ([K1, Corollary 4$])$.

$$
J_{P}=\mathbb{C}^{n} \backslash\left\{z \in \mathbb{C}^{n}: \lim _{j \rightarrow \infty}\left|P^{j}(z)\right|=\infty\right\} .
$$

\section{Proof of the MAIN Result}

Proof of Theorem 1.2. In view of Theorem 1.1 $J_{P}$ is compact, thus we can fix such an $r>1$ that $J_{P} \subset B_{r}:=\left\{z \in \mathbb{C}^{n}:|z| \leq r\right\}$. Then by (2.3) we get

$$
P^{j}\left(J_{P}\right) \subset B_{r}, \quad \text { if } j \in \mathbb{N}_{0} .
$$

We define $A \geq \delta$ and $\alpha \in(0,1]$ as follows:

$$
A:=\max \left\{\delta, \max _{|z| \leq 2 r}\left|P^{\prime}(z)\right|\right\} \quad \text { and } \quad \alpha:=\log _{A} \delta .
$$

If $z$ is in $J_{P}$, we have $V_{J_{P}}(z)=\operatorname{dist}\left(z, J_{P}\right)=0$, and inequality (HCP) is obvious.

Now let $z$ be in $\mathbb{C}^{n} \backslash J_{P}$ with $|z|<2 r$. Since $J_{P}$ is compact, there exists a $z_{0} \in J_{P}$ such that $\left|z-z_{0}\right|=\operatorname{dist}\left(z, J_{P}\right)>0$. If $w \in\left[z_{0}, z\right]:=\left\{(1-t) z_{0}+t z:\right.$ $t \in[0,1]\}$ and $w \neq z_{0}$ then $w$ does not belong to $J_{P}$.

We pick $k=k(z)$ in $\mathbb{N}$ to satisfy the following conditions:

$$
\left|P^{j}(w)\right| \leq 2 r \quad \text { for each } w \in\left[z_{0}, z\right] \text { and } 0 \leq j \leq k-1,
$$

and

$$
\text { there exists a } z_{1} \in\left[z_{0}, z\right] \text { with } \quad\left|P^{k}\left(z_{1}\right)\right|>2 r \text {. }
$$

Note that we can find such a point according to Lemma 2.2.

Let $w \in\left[z_{0}, z\right]$ be given. By the definition of $A$ we conclude that

$$
\begin{aligned}
\left|\left(P^{k}\right)^{\prime}(w)\right| & =\left|P^{\prime}\left(P^{k-1}(w)\right) \circ \ldots \circ P^{\prime}(P(w)) \circ P^{\prime}(w)\right| \\
& \leq\left|P^{\prime}\left(P^{k-1}(w)\right)\right| \ldots\left|P^{\prime}(w)\right| \leq A^{k}
\end{aligned}
$$

since $\left|P^{k-1}(w)\right|, \ldots,|P(z)|,|z| \leq 2 r$ from (3.2). Hence integration along $\left[z_{0}, z_{1}\right]$ gives $\left|P^{k}\left(z_{0}\right)-P^{k}\left(z_{1}\right)\right| \leq A^{k}\left|z_{0}-z_{1}\right|$. Combining this with (3.1) and (3.3) we obtain $2 r<\left|P^{k}\left(z_{1}\right)\right| \leq A^{k}\left|z_{0}-z_{1}\right|+\left|P^{k}\left(z_{0}\right)\right| \leq A^{k} \operatorname{dist}\left(z, J_{P}\right)+r$, since $\left|z_{0}-z_{1}\right| \leq\left|z_{0}-z\right|=\operatorname{dist}\left(z, J_{P}\right)$. This gives $A^{k} \operatorname{dist}\left(z, J_{P}\right) \geq r>1$ by the choice of $r$. Consequently by the definition of $\alpha$ we get

$$
\left[\operatorname{dist}\left(z, J_{P}\right)\right]^{\alpha}>\left(A^{-k}\right)^{\alpha}=\delta^{-k} .
$$

In view of (2.3) and (2.2) we obtain

$$
V_{J_{P}}(z)=V_{P-k\left(J_{P}\right)}(z) \leq \delta^{-k} V_{J_{P}}\left(P^{k}(z)\right) .
$$

We define now $C:=\sup \left\{V_{J_{P}}(P(w)):|w| \leq 2 r\right\}$. Note that $C$ is a finite number, because the polynomial mapping $P$ is continuous and by Theorem 1.1 the $L$-extremal function $V_{J_{P}}$ is also continuous. From (3.2) we get $\left|P^{k-1}(z)\right| \leq 2 r$, whence $\left|V_{J_{P}}\left(P^{k}(z)\right)\right| \leq C$. Thus in view of (3.4) and (3.5)

$$
V_{J_{P}}(z) \leq C\left[\operatorname{dist}\left(z, J_{P}\right)\right]^{\alpha} .
$$

Moreover, if $\operatorname{dist}\left(z, J_{P}\right) \leq 1$, then $|z| \leq \operatorname{dist}\left(z, J_{P}\right)+\left|z_{0}\right| \leq 1+r \leq 2 r$ and this completes the proof of our assertion. 


\section{ACKNOWLEDGMENTS}

The author wishes to express her thanks to Professor Wiesław Pleśniak for his advice and help.

\section{REFERENCES}

[CG] L. Carleson, T. W. Gamelin, Complex Dynamics, (Springer, New York, 1993). MR 94h:30033

[E] A.Edigarian, Remarks on Hölder Continuity Property of $L$-regular sets, (personal communication).

[FS] J. E. Fornaess, N. Sibony, Complex Hénon mappings in $\mathbb{C}^{2}$ and Fatou-Bieberbach domains, Duke Math. Journal 65 (1992), 345-380. MR 93d:32040

[K1] M. Klimek, Pluricomplex Green functions for filled-in Julia sets in $\mathbb{C}^{n}$, (preprint, University College Dublin, 1991).

[K2] M. Klimek, Pluripotential Theory, (Oxford University Press, 1993).

[K3] M. Klimek, Metrics associated with extremal plurisubharmonic functions, Proc. Amer. Math. Soc. 123(9) (1995), 2763-2770. MR 95k:32015

[M] A. A. Markov, On a problem posed by D. I. Mendeleev, Izv. Akad. Nauk. St-Petersbourg 62 (1889), 1-24 (in Russian).

[PP] W. PAwŁucki, W. PleŚniak, Markov inequality and $C^{\infty}$ functions on sets with polynomial cusps, Math. Ann. 275(3) (1986), 467-480. MR 87k:32031

[P] W. PleŚnIAK, Compact subsets of $\mathbb{C}^{n}$ preserving Markov's inequality, Mat. Vesnik 40 (1988), 295-300. MR 91e:32011

[RS] Q. I. Rahman, G. Schmeisser, Les inégalités de Markoff et de Bernstein, (Les Presses de l'Université de Montréal, 1983). MR 85f:41009

[S1] J. SiCIAK, Degree of convergence of some sequences in the conformal mapping theory, Colloq. Math. 16 (1967), 49-59. MR 35:4385

[S2] J. Siciak, Extremal plurisubharmonic functions in $\mathbb{C}^{n}$, Ann. Polon. Math. 39 (1981), 175-211. MR 83e:32018

Institute of Mathematics, Jagiellonian University, ul.Reymonta 4, 30-059 Kraków, POLAND

E-mail address: kosek@im.uj.edu.pl 